\title{
Modeling reactive magnetron sputtering: opportunities and challenges
}

\author{
D. Depla, K. Strijckmans, A. Dulmaa, F. Cougnon, R. Dedoncker, R. \\ Schelfhout, I. Schramm, F. Moens, R. De Gryse \\ Department of Solid State Sciences, Ghent University, Krijgslaan 281 (S1), 9000 Gent, \\ Belgium
}

\begin{abstract}
The complexity of the reactive magnetron sputtering process is demonstrated by four simulation examples. The examples, commonly encountered during the application of this process for thin film deposition, are described by a numerical model for reactive sputter deposition. A short description of the current model precedes these case studies. In the first example, redeposition of sputtered atoms on the target is studied by its effect on the hysteresis behavior often observed during reactive sputtering. Secondly, the complexity of current-voltage characteristics during reactive magnetron sputtering is treated. The influence of substrate rotation and the pulsing of the discharge current illustrate the time dependence of the reactive sputtering process. As a conclusion, the two main challenges for a further improvement of the model are discussed.
\end{abstract}

Keywords: reactive magnetron sputtering, modeling 
1

2

3

$4 \quad$

5

6

7

8

9

\section{Introduction}

Modeling of reactive magnetron sputtering is essential to get a full understanding of this process. This bold statement is based on the long experience of our team supported by many researchers in the thin film communities. Or stated by J.E. Greene in one of his review papers on the history of thin film deposition [1], "Another important recent accomplishment in sputter deposition is the evolutionary development of very useful models, which are increasingly more accurate, of the highly complex reactive-sputtering process.", and further in the same paper "...their value is in allowing the researcher to pose "what if" questions before initiating experiments, thus greatly decreasing the number of iterations prior to achieving desired results." Inspired by this latter statement, a few, rather complex, examples of simulations performed with the RSD model $[2,3]$, in combination with the Monte Carlo particle trajectory code SIMTRA $[4,5]$, will be discussed. These examples will not only demonstrate the opportunities for other researchers to apply the model to specific problems, but they will also show the complexity, and hence the challenges for future improvement in modeling reactive magnetron sputtering. The input files for the simulations presented in this paper are available on [6]. The paper starts with a short description of the RSD model, and consequently discusses the influence of redeposition, the behavior of I-V characteristics during reactive sputtering, substrate rotation, and the impact of current pulses on the reactive sputtering process. 


\section{Reactive sputtering and the RSD model}

The key elements of reactive sputtering, and the RSD model are described in this section. Reactive magnetron sputter deposition is a complicated process which can puzzle the less experienced researcher. To assist the understanding of this short description (and the remainder of the paper), the interested reader can benefit from a tutorial paper on this topic [7]. Technical details about the model implemented in the RSD software can be found in previous published work $[8,2,3]$.

The RSD model follows the tradition in modeling reactive sputtering to describe this process based on the conservation of reactive species [9]. To simplify the discussion, only diatomic gases such as oxygen and nitrogen are considered. The molecular reactive gas enters the vacuum chamber at a reactive gas flow equal to $Q_{i n}$. The initial gas mainly reacts with the deposited material to form the desired compound on the substrate. The consumption rate of the reactive gas atoms in this process is described by $Q_{c}$. The reactive gas reacts to a minor extent also at the target. Again a consumption rate $Q_{t}$ is defined. When reactive gas flow remains lower than a given reactive flow, known as the first critical point $Q_{i n, 1}$, this description is basically sufficient to explain the observed features of the process. Indeed, if the deposition rate remains high, the reactive gas partial pressure is low, and other deposition parameters hardly differ from the condition without reactive gas addition. Therefore, this regime of the process is known as the metallic mode. The deposited compound in this regime is typically substoichiometric.

At the first critical point, the reactive gas flow is balanced by the maximum consumption rate, or the getter capacity, of the process. Further reactive 
gas addition leads to important process changes: an increase of the reactive gas partial pressure, and a drop in the deposition rate. The origin of these changes is the interaction of the reactive gas with the target which leads to compound formation. The lower sputter yield of the target under these conditions reduces the gas getter capacity as less metal is deposited. Hence, the reactive gas partial pressure increases until a new steady state condition is reached where the target is fully covered by a compound layer. Under these conditions, known as the compound or poisoned mode, a substantial fraction of the reactive gas is consumed by the vacuum pump, or the consumption rate $Q_{p}$ of the pump is high. Due to the difference in sputter yield between the metallic and the compound mode, it is not possible to return to the metallic mode at the same reactive flow of the first critical point, and it is necessary to reduce the flow to a lower value. The flow at which the process switches back to the metallic mode is known as the second critical point $Q_{i n, 2}$. The transition for metallic to poisoned mode (and reverse) occurs quite often abrupt. So, when the reactive gas flow is set slightly higher (lower) than the first (second) critical point a jump in the process parameters is observed which explains the definition of these critical points. As the two critical points do not coincide, a hysteresis is observed for the main process parameters as a function of the reactive gas flow. The quantitative description of this hysteresis is the main objective of the RSD model.

So, essentially, the RSD model describes the balance between the gas input, and the consumption rates of the pump, the substrate, and the target,

$$
Q_{i n}=Q_{p}+Q_{c}+Q_{t}
$$


The easiest way to grasp the essence of the model is a short description of how these rates are approached. Both the consumption rate by the vacuum pump and the deposited material can easily be understood. The first is defined by the reactive gas partial pressure, and the pumping speed of the deposition set-up for the reactive gas. The consumption rate by the deposited material is described as an incorporation process of the reactive gas molecules. The efficiency of the process is defined by a material dependent incorporation, or sticking, coefficient. The consumption rate is further defined by the flux of the reactive gas molecules towards the deposited material, and the metal fraction of the deposited material. The latter is defined by the deposition rate of both metal and compound which depend on the target condition. To describe the deposition rate at different locations in the deposition setup, the RSD model can use the in-house build particle trajectory code SIMTRA. A short description of this code will be given further in the text. Up to this point, the RSD model builds on the contribution of several authors to the field. The model distinguishes itself by the description of the consumption rate by the target as a consequence of several target processes. Evidently sputtering of metal and compound molecules is the first one. The chemisorption of reactive gas molecules at the target surface is described in a similar fashion as the chemical reaction between the reactive gas and the deposited material. The implantation of the reactive gas into the target is treated in a distinctive way for the RSD model. The chemical reaction of the implanted reactive gas atoms is treated as a second order chemical reaction. By explicitly describing this reaction, the model predicts a fraction of non-reacted gas atoms in the target. Although no direct evidence can be given for their presence, there is 
substantial experimental indirect evidence such as discharge voltage changes $[10,11]$, time dependent sputter yields [12], and the observation of a second hysteresis [13]. The latter is attributed to a change of the target state due to the presence of these non-reacted atoms. Finally, the RSD model can also account for the redeposition of sputtered atoms on the target. A nonuniform current profile on the target, a deposition profile on the substrate, and a dependence of the current-voltage discharge characteristic on the target condition, has made the model a versatile tool to investigate reactive magnetron sputter deposition. As mentioned before, the deposition rate at different locations in the deposition setup can be modeled with SIMTRA. It is a test particle Monte Carlo code which simulates the trajectory of sputtered atoms from the target towards their final location. Test particles are launched from the target with an energy and a direction randomly selected from the nascent energy and angular distribution. The collisions with the sputter gas atoms are described based on spherical symmetric interaction potential. The test particle is followed until it arrives on any of the predefined surfaces which describe the experimental set-up. More details on this code can be found in $[4,5]$. Both SIMTRA and RSD have been implemented in a downloadable executable[14]. The focus of the current paper is to illustrate the possibility of the RSD model with several interesting examples for the thin film community.

\section{Example 1: Redeposition of sputtered atoms}

Atoms sputtered from the target travel through the gas phase towards their final landing place. They can collide with the gas atoms, and recoil onto 
the target. This process is known as redeposition. To study redeposition, the particle trajectory code SIMTRA is one of the possible tools. Figure 1 shows the fraction of the sputtered atoms that returns to the target as a function of the argon pressure in the vacuum chamber. The return probability for elements lighter than argon $(\mathrm{Li}, \mathrm{Al})$ is much larger than for heavier elements. This can be understood from binary collision physics. The deflection angle of the sputtered atoms increases smoothly as function of the ratio between the masses of the gas atom and the sputtered atom [15]. The influence of the argon pressure can be understood from the increased collision probability when the gas density between ejection and deposition position is increased.

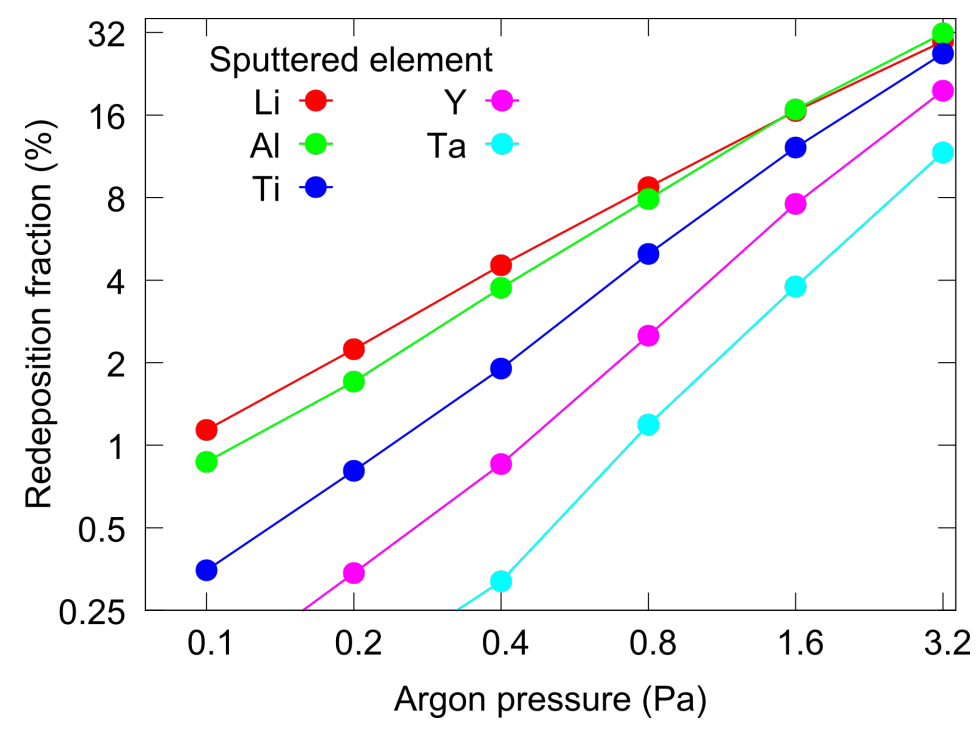

Figure 1: The simulated redeposition fraction on the target as a function of the argon pressure for different elements. Simulation conditions are a $30 \times 30 \times 30 \mathrm{~cm}^{3}$ chamber with a two inch magnetron where the target surface is positioned in the center and $7.5 \mathrm{~cm}$ away of one of the chamber walls. A $2.5 \times 2.5 \mathrm{~cm}^{2}$ substrate was positioned parallel with the target surface at a mutual distance of $10 \mathrm{~cm}$. The total number of simulated particles was set to $10^{5}$. 
At higher pressures, and especially for light elements, the redeposition fraction can become substantial which explains the necessity to include redeposition, next to chemisorption, reactive ion implantation, and sputtering, as the fourth target process in the description of the target condition during reactive sputtering [16]. Figure 2 shows the simulated influence of the redeposition fraction on one of the typical studied process curves, i.e. the reactive gas partial pressure as a function of the reactive gas flow. The simulation shows that the first critical point, i.e. the transition point from the low oxygen pressure to the high oxygen pressure regime, shifts towards lower oxygen flows while the second critical point remains unaffected. This behavior could have multiple explanations. When the redeposition fraction is increased, less material is deposited on the different surfaces of the vacuum chamber. This reduces the getter capacity, and hence less oxygen needs to be introduced to switch from metallic mode to poisoned mode. Once in poisoned mode, the gas consumption rate $Q_{c}$ is a negligible part of the total gas consumption rate, and hence the lower deposition rate does not influence the second critical point as observed from the simulations. This explanation is consistent with the result of an experimental study on facing target sputtering [17] which shows a clear shift of the first critical point while a minor shift of the second critical point. An alternative explanation could be based on the lowering of the effective erosion rate of the target, i.e. the erosion rate due to sputter bombardment reduced by the growth rate by redeposition. A change of only the effective erosion rate can easily be implemented in the simulations by changing the effective sputter yield of both the compound and metal. 

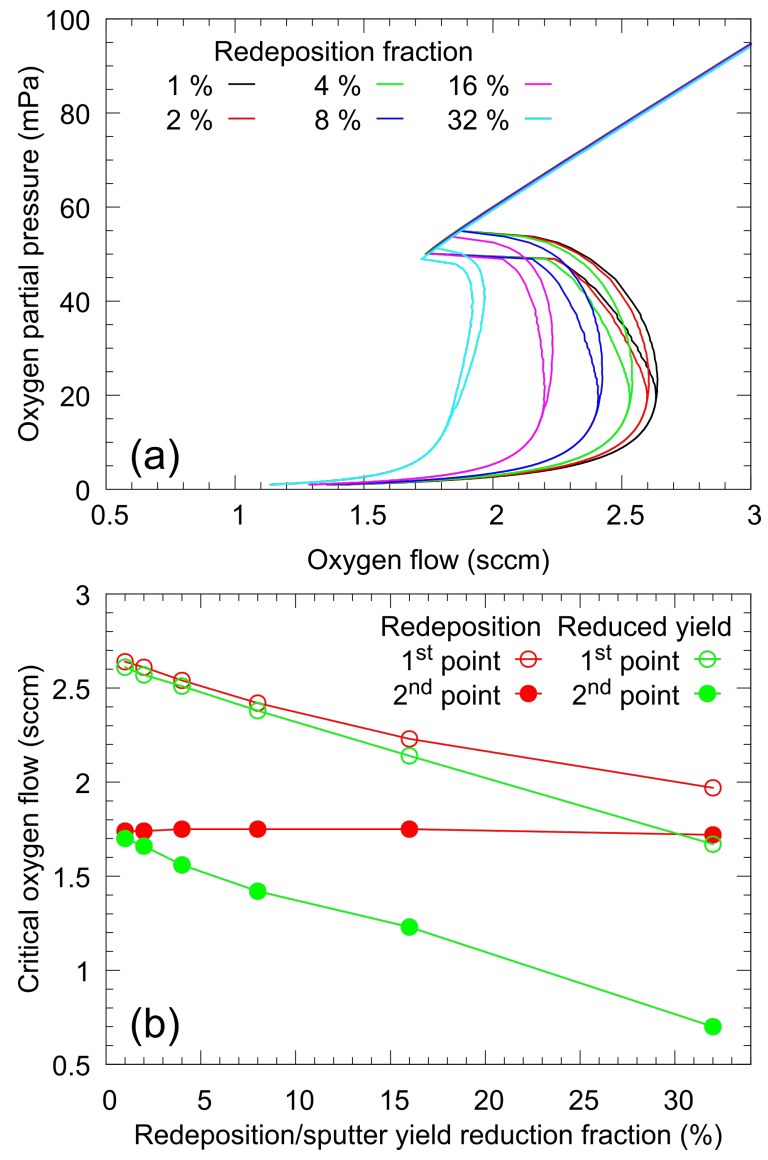

Figure 2: (a) The simulated oxygen partial pressure as a function of the oxygen flow for different levels of redeposition. The same chamber configuration as used for Figure 1 was implemented in SIMTRA, and the output was used in the RSD software. An Al target was used. The same simulated redeposition profile at $0.4 \mathrm{~Pa}$ was used, to allow a straightforward interpretation of the influence of the redeposition fraction on the different hystereses. All other parameters remained fixed. The smoother transition at the first critical point as compared to the second critical point is mainly to the gradual oxidation of both substrate and target. (b) The critical oxygen flow as a function of the redeposition fraction (red markers), or the sputter yield reduction fraction (green markers). The first critical point is indicated with open markers while the second critical point is represented by closed makers. The simulation conditions were identical apart from the shown dependency. 
This will affect the getter capacity, and results in a similar shift of the first critical point as shown in Figure 2b. However, a clear difference for the second critical point is observed. When the effective sputter yield is reduced, the second critical point shifts in a similar manner as the first critical point towards lower oxygen flows. The behavior of the second critical point under these conditions can be explained as follows. The second critical point is mainly defined by the chemical reaction of the implanted oxygen in the target. When the effective sputter yield is reduced, the reaction time of the implanted species becomes longer which results in a higher target oxidation state. To return to the metallic mode, the oxygen fraction in the discharge must be reduced to a lower value, or stated differently the oxygen flow at the second critical point must be lower. This effect has no influence when the redeposition fraction is increased. Indeed, in the current version of the RSD code, as compared to the version used in [17], compound sputtering is described as an atomistic process while the congruent aspect of sputtering has been preserved. When the reactive atoms are redeposited on the target, their reaction probability is low as the target surface at the second critical point is fully poisoned. The redeposited metal atoms will only influence to a minor extend the erosion rate as their sputter yield is high. Indeed, the redeposited metal is incorporated at the surface as a non-reacted metal. The rather low oxygen sticking probability of 0.1 used in the simulations results in a lower probability for the reaction of the redeposited metal by chemisorption as compared to the probability to be re-sputtered with a high yield as non-reacted metal.

In summary, based on these simulations it is clear that redeposition can 
be an important effect during magnetron sputter deposition, and its impact on the first critical point can be understood from a reduced deposition rate that lowers the getter capacity of the process.

\section{Example 2: I-V characteristics}

A now less common approach to study reactive magnetron sputtering is sweeping the discharge current while the reactive gas flow is maintained. Different process parameters, such as the reactive gas partial pressure or the discharge voltage, as function of the discharge current can be measured. When the discharge voltage is tracked, the obtained plot is known as an I-V characteristic which presents the applied discharge current as a function of the measured discharge voltage. Figure 3 depicts two possible shapes of a I-V characteristic. The top figure (a) represents a simulated I-V characteristic for Ti measured in a mixture of $\mathrm{O}_{2} / \mathrm{Ar}$, while the bottom figure (b) shows the same information for Al. The simulation of this kind of I-V characteristic requires experimental input of the $\mathrm{I}-\mathrm{V}$ characteristics in metallic and poisoned mode. When this information is accessible, the RSD model permits to calculate the entire characteristic based on the assumption that the ion induced electron yield is a weighted function of the electron yield of the oxide and the metal. More details on this approach can be found in Strijckmans et al. [7], and in Depla et al. [18] In this context, it is important to mention that in the $\mathrm{Ti} / \mathrm{O}_{2}$ case the simulated curve is an approximation to illustrate the different behavior between $\mathrm{Ti}$ and $\mathrm{Al}$. Although, the general trend is correctly simulated, the RSD model does not account for the presence of different titanium oxides. 

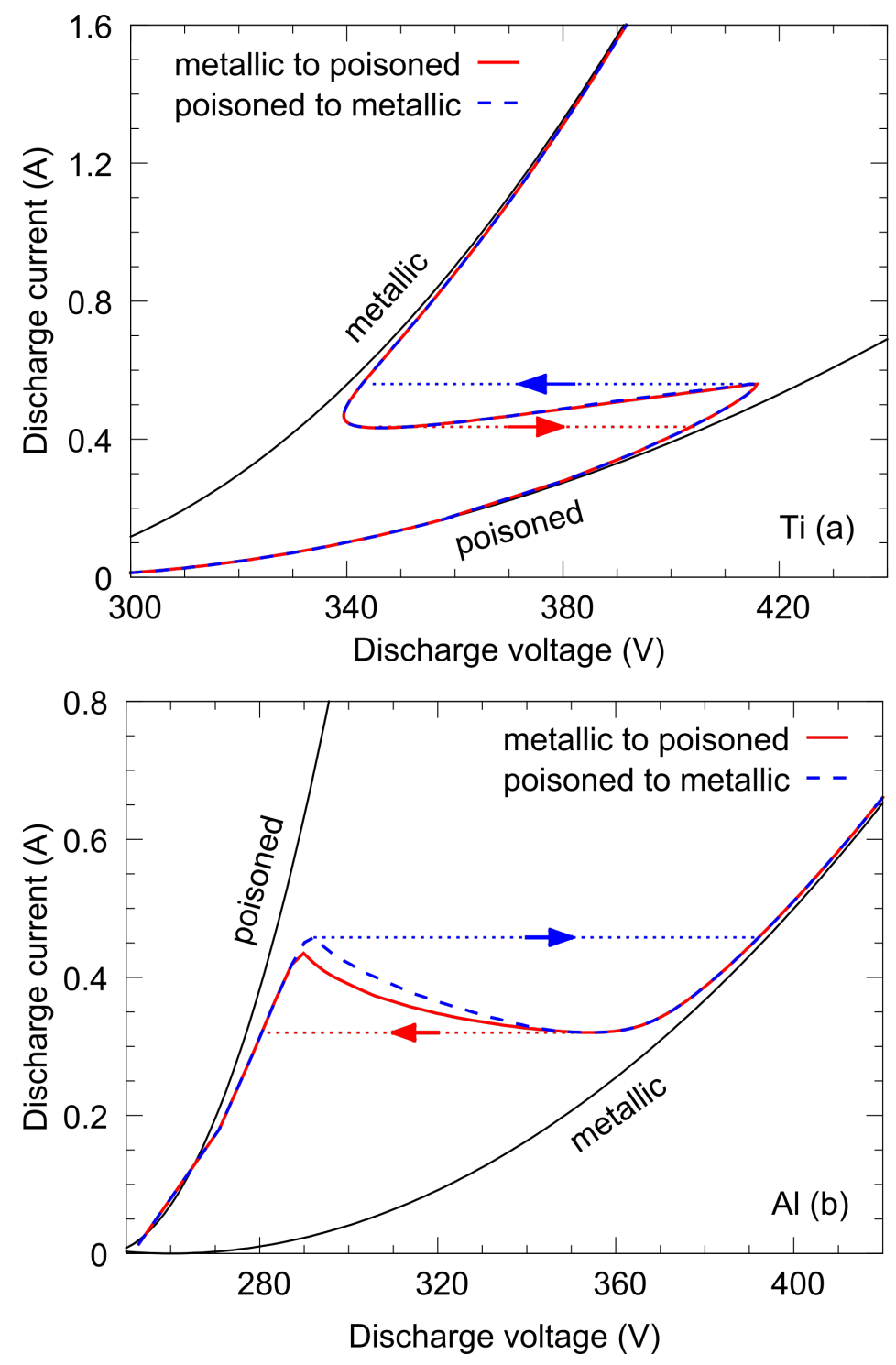

Figure 3: Simulated I-V characteristics for (a) $\mathrm{Ti}$ and (b) $\mathrm{Al}$ in a $\mathrm{O}_{2} / \mathrm{Ar}$ atmosphere. The oxygen flow was fixed at $1.5 \mathrm{sccm}$. To describe the I-V characteristic in the transition zone, the I-V characteristics of a metal target and a fully poisoned target (black lines) are combined. This combination is based on a weighted average of the electron yields which depends on the target surface fractions. In the case of $\mathrm{Al}$ the $\mathrm{I}-\mathrm{V}$ characteristic is doubled valued in the transition region, while this is not the case for Ti. The main reason for this difference is the larger ratio between the metal and the oxide sputter yield in the case of $\mathrm{Al}$. 
At low discharge current(power), the target is in poisoned mode. When the current is increased, the target will abruptly change from the poisoned state to the metal state as indicated by the dashed lines. The main difference between $\mathrm{Al}$ and $\mathrm{Ti}$ is the direction of the transition. In the case of $\mathrm{Al}$, the discharge voltage increases when the target changes to the metallic state, while for Ti the opposite occurs. This has an important effect on the process control. With the available power supplies for DC magnetron sputtering, it is possible to stabilize the discharge on current, voltage or power. The simulations shows that for both $\mathrm{Al}$ and $\mathrm{Ti}$ it is impossible to reach the transition region between the metallic and the poisoned state when the discharge is current controlled. The target condition will abruptly change as indicated by the dashed lines. In the case of $\mathrm{Al}$, the simulated $\mathrm{I}-\mathrm{V}$ characteristics is a double valued function of the discharge voltage, in contrast to a triple valued function for the Ti case, which permits to perform voltage-controlled experiments. The implementation of the I-V characteristic of a magnetron discharge in metallic and poisoned mode in the RSD code permits to describe the behavior of the discharge current and voltage at fixed oxygen and argon flows. Also the change of the oxygen partial pressure can be calculated under these conditions. This permits to compare the simulation results with experiments that are not influenced by long term effects. Indeed, in a "classical" hysteresis experiment as shown in Figure 2a, the oxygen flow is stepwise increased, and the deposition parameters are registered. As the stabilization time after an oxygen flow change is in the order of minutes, the measurement of a full process curve requires quite some time. Long term effects such as target erosion can in this way influence the measurement, and hence its in- 
terpretation. By scanning I-V characteristics at different, but non-sequential flows, this problem can be circumvented, and permits to investigate reactive magnetron sputter deposition in an alternative way. . An example of this kind of experiments is presented in Figure 4. This kind of measurements (and simulations) have shown to be essential to understand the poisoning behavior of the target [13].

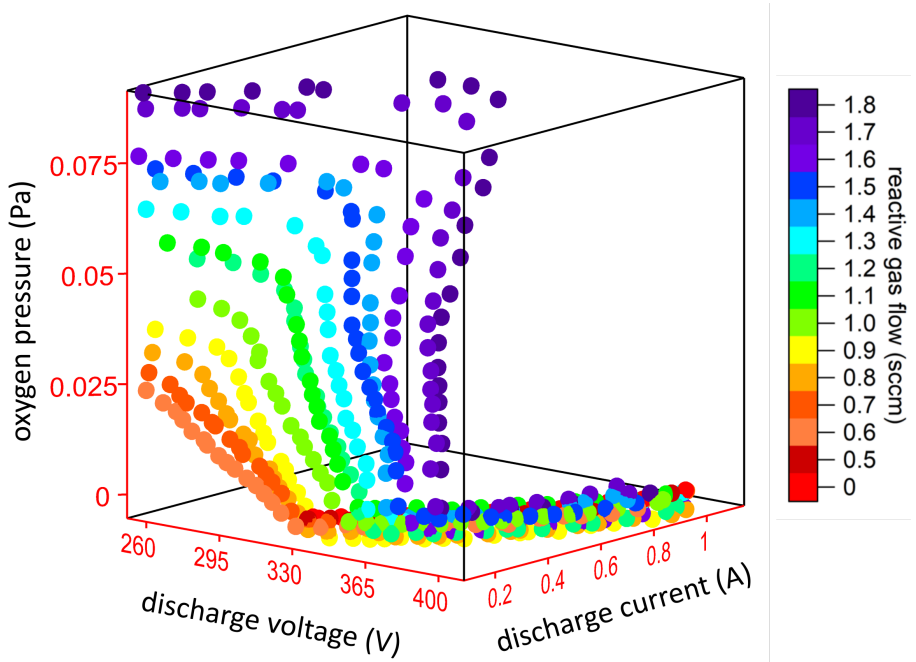

Figure 4: Measurement of the current, voltage, pressure and flow space during reactive magnetron sputtering of $\mathrm{Al}$ in a $\mathrm{O}_{2} / \mathrm{Ar}$ mixture. Experimental conditions are a 2 inch target at an argon pressure 0.4 Pa. More experimental details can be found in Schelfhout et al. [13]

\section{Example 3: Sample rotation}

The gas distribution of both the reactive and the sputter gas are important to improve film uniformity, especially for large area coaters [19]. The reactive gas distribution can affect in a complex way the film properties [20] 
and target poisoning [21]. The influence of the reactive gas distribution can only be modeled with more advanced codes that include the gas dynamics $[22]$.

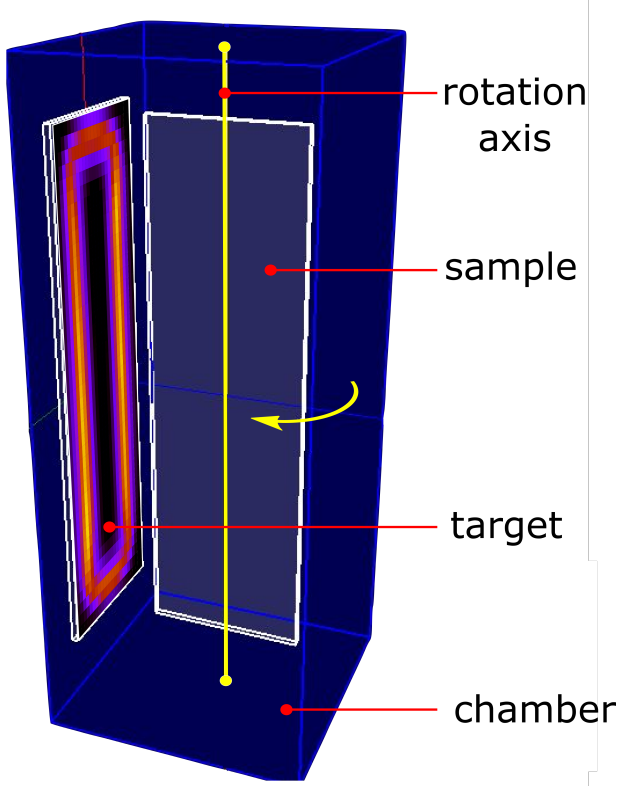

Figure 5: Overview of the implemented setup to investigate the influence of sample rotation on the hysteresis behavior. A large substrate $\left(40 \times 12 \mathrm{~cm}^{2}\right)$ is placed in a vacuum chamber with dimensions $20 \times 20 \times 50 \mathrm{~cm}^{3}$ (dark blue). The target $(\mathrm{Al})$ is a rectangular plate with the same size as the substrate. The racetrack of the rectangular target is an experimental measured erosion profile where the used resolution is $1 \times 0.5 \mathrm{~cm}^{2}$. When the substrate is parallel to the target the distance between them is $10 \mathrm{~cm}$. SIMTRA simulations were performed at $0.4 \mathrm{~Pa}$ argon. For the RSD simulations the discharge current was set at $2 \mathrm{~A}$ and the pumping speed equaled $55 \mathrm{l} / \mathrm{s}$.

Due to the increased complexity, one often needs to find a compromise to obtain reasonable simulation times, e.g. a less detailed description of the 
target poisoning mechanisms. In the RSD code, the gas distribution is not included as it is assumed that the oxygen pressure is uniform over the vacuum chamber. The distribution of the sputtered atoms is however implemented including the output of SIMTRA simulations. This permits to investigate the influence of sample rotation as an alternative to optimize film uniformity. When the substrate orientation affects in a major way the deposition profile, it can be expected that the rotation speed of a substrate will influence the reactive sputter process. This point will be discussed in more detail based on the setup shown in Figure 5. RSD simulations were performed for different rotation speeds of the substrate. When the rotation speed is increased the first critical point shifts towards higher oxygen flow, while the second critical point remains unaffected (Figure 6a). To understand this behavior it is instructive to follow the dynamic behavior of the oxygen partial pressure (Figure 6b). When the substrate is parallel to the target, the oxygen pressure is higher as compared to the situation when the substrate stands perpendicular to the target as in Figure 5. This indicates that less oxygen is consumed during the parallel substrate orientation. The influence of the substrate orientation lays in the blocking of the deposition on the chamber walls which is low when the substrate is parallel with the target (parallel in Figure 6a) and high when the substrate is perpendicular positioned towards the target (perpendicular in Figure 6a). Or stated differently, a larger effective deposition area is obtained as substantial deposition on the chamber walls is permitted when the substrate is in the perpendicular position. 

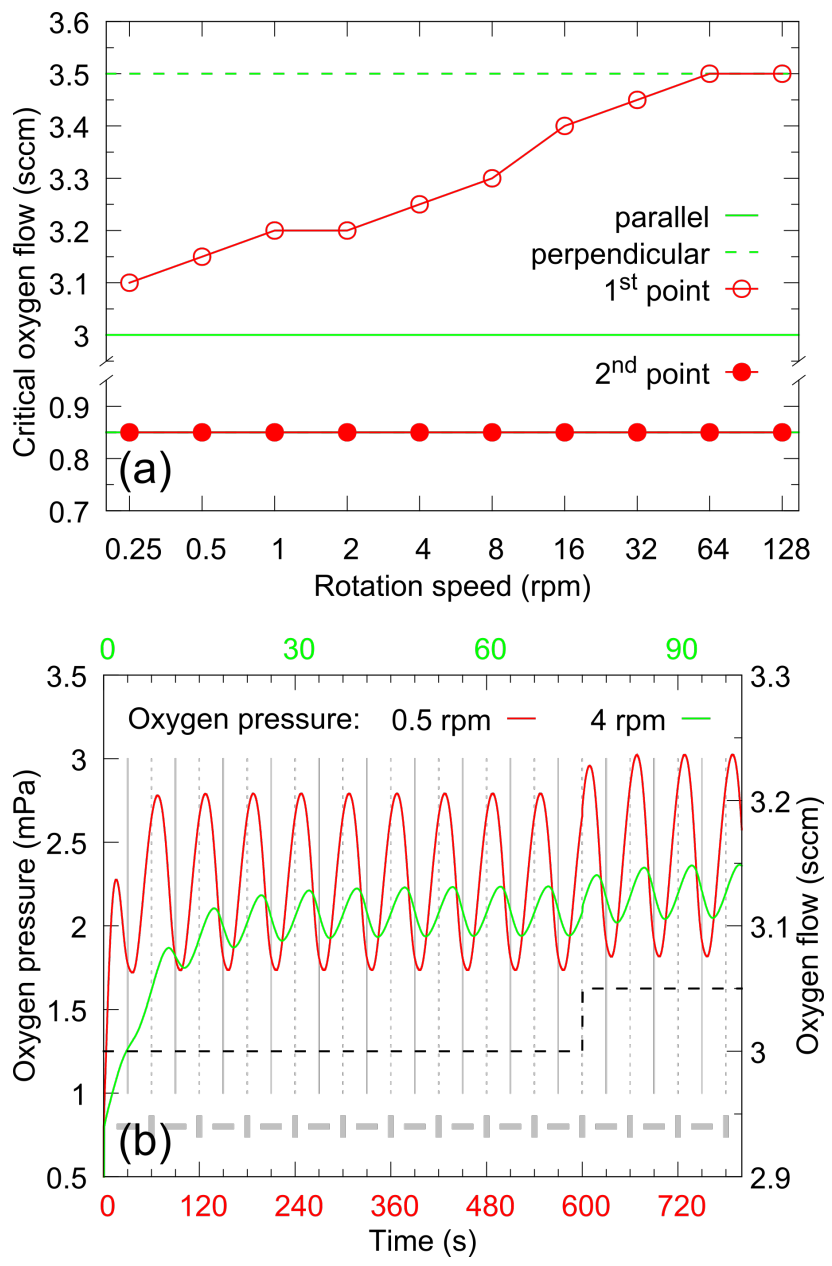

Figure 6: (a) The first (open markers) and second (closed markers) critical point as a function of the substrate rotation speed in rounds per minute (rpm). The first critical point calculated for two situations with a stationary substrate parallel to the target (full green line) and perpendicular to the target (dashed green line) are also indicated. (b) The time dependency of the oxygen pressure (left axis) when the substrate rotates at $0.5 \mathrm{rpm}$ (lower axis) and $4 \mathrm{rpm}$ (upper axis) in metallic mode. The value of the oxygen flow is indicated on the right axis. The grey vertical lines indicate when the substrate is parallel (dashed line) or perpendicular (full line) to the target. These positions of the substrate are indicated by the grey blocks in lower part of the figure. 
From this reasoning it can also be understood that the influence of the chamber size will be minor as in the simulation the wall surface area is already sufficiently large to permit a sufficient spread of the sputtered material. The influence of the chamber size will mainly influence the maximal value of the first critical point. The lower first critical oxygen flows at low rotation speeds is the result of larger fluctuations in the oxygen pressure. In this way the critical pressure to induce the transition from metallic to poisoned mode is easier accessed. By increasing the rotation speed, the average getter capacity increases which requires higher oxygen flows to poison the process. The above reasoning is confirmed by two simulations with a stationary substrate: a parallel and a perpendicular substrate. The first critical point gradually changes from the low value for the parallel configuration (full green line in Figure 6a) to the high value for the perpendicular configuration (dashed green line in Figure 6a). The simulations also show that the second critical point is not affected by the sample rotation. Indeed, the return from poisoned mode is solely defined by target processes.

\section{Example 4: Pulsing the discharge current}

In some cases, such as to avoid arcing, it can be beneficial to use a pulsed current instead of a direct current. As target poisoning occurs at a time scale of 0.1 to 10 seconds, it can be expected that at high frequencies no differences in the hysteresis behavior is observed. This can indeed be observed in Figure 7a. Above a frequency of $20 \mathrm{~Hz}$ the first and second critical point almost coincide with the points simulated for the DC case $(0 \mathrm{~Hz})$. In the simulation, the change of the discharge voltage with the changing discharge current 
is included. This results in a minor increase of the average sputter yield for the pulsing current simulations as compared to the DC case, which explains the small difference between the critical points at high current pulsing frequencies. At low frequency, no hysteresis is observed as the two critical points coincides. This is in agreement with the experiments by Billard et al. [23, 24, 25] who demonstrated that hysteresis can be avoided by pulsing the discharge current at low frequencies. The elimination of the hysteresis can be understood as follows. If the oxygen flow is high enough, the target surface oxidizes completely at low current, but is completely cleaned again in the high current regime of the same cycle. At intermediate frequencies, this is not possible anymore, and the hysteresis starts to widen. When the target condition is followed in time at a frequency within this intermediate regime, the complexity of the poisoning mechanism becomes visible. This is illustrated in Figure 7b which shows the time dependency of the average chemisorbed fraction $\bar{\theta}_{c}$ and the average oxide fraction $\bar{\theta}_{r}$ together with the ion current (right axis). The chemisorbed fraction is defined as the target compound fraction formed by chemisorption of oxygen molecules. Oxygen is also implanted into the target. Due to the target erosion the implanted oxygen travels towards the target surface, and as discussed before, it can react with the target material during this journey to form compound. The average target fraction covered by the oxide is given by $\bar{\theta}_{r}$. When the discharge current increases, it is expected that the compound layer is sputtered, and both fractions starts to decrease. This occurs however not immediately. When the discharge current is zero, the implanted compound can further react with the target material, slightly increasing the oxide fraction. 

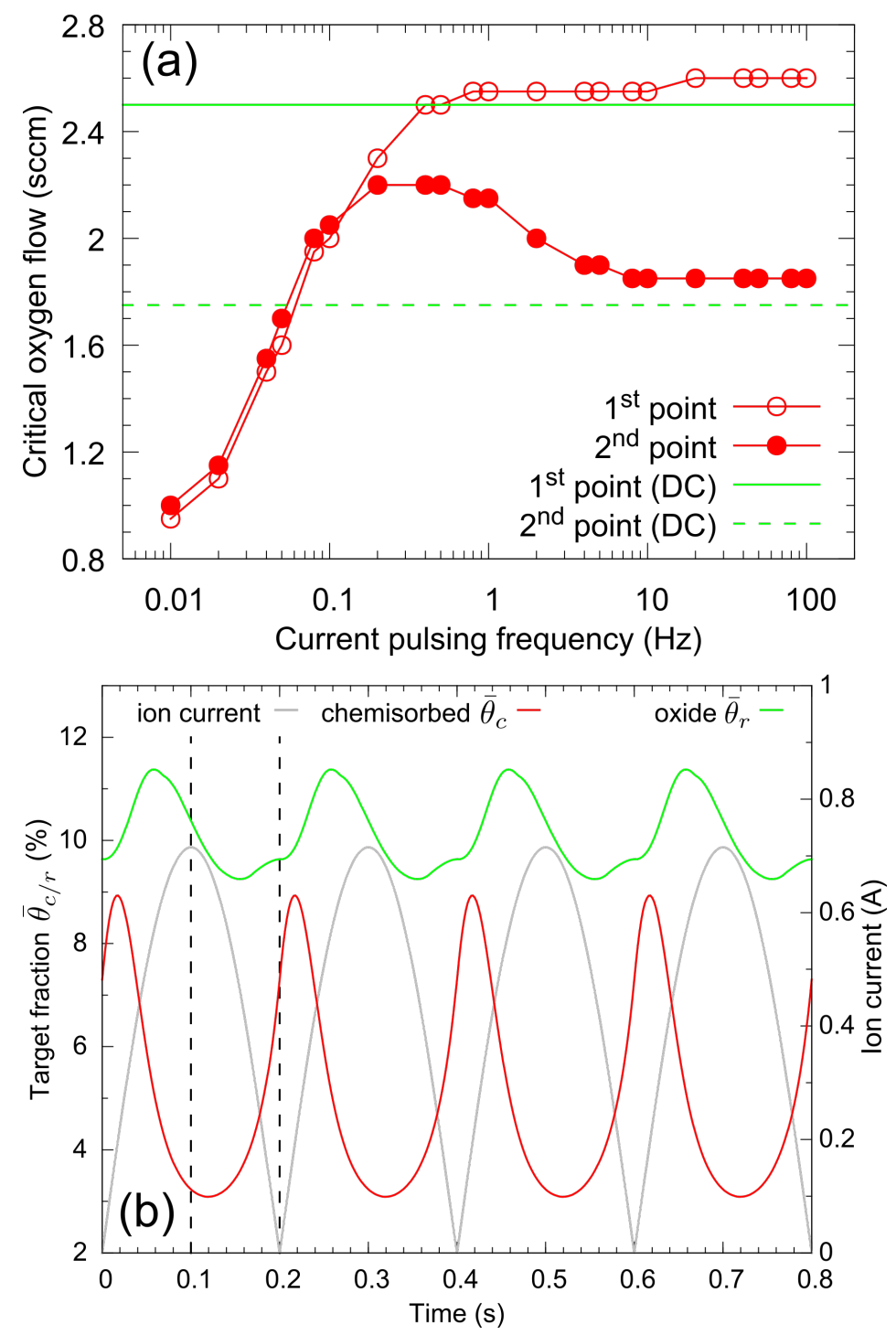

Figure 7: (a) The first (open markers) and second (closed markers) critical point as a function of the current pulsing frequency. The green lines indicate that first and second critical point for direct current (DC) conditions. (b) The time dependency of the average chemisorbed fraction $\bar{\theta}_{c}$ and the compound fraction $\bar{\theta}_{r}$. On the right hand axis the ion current to the target is shown. The current pulsing frequency was set to $5 \mathrm{~Hz}$. The simulations were performed at an oxygen flow of $2.5 \mathrm{sccm}$, i.e. just before the first critical point. The simulation were performed for a two inch planar cylindrical aluminum target. 
Also, oxide will be formed deeper in the target. When the discharge current increases, the formed oxide travels towards the surface, and increases the oxide fraction $\theta_{r}$. As the reactive gas cannot chemisorb onto the oxide fraction, its fraction will also decrease. The decrease will occur faster due to sputtering. Nevertheless, due to the implantation, and the formation of an oxide layer thicker than one monolayer, the target condition is not in phase with the modulation of the discharge current. Although there is currently no experimental evidence for this behavior, this behavior shows once more the complexity of reactive magnetron sputtering.

\section{Conclusion}

Despite the improvements in understanding reactive sputtering by the development of the RSD model, there is still a long way in going beyond the "what if" questions. Two main obstacles need to be taken. The first is a further mapping, and implementation of other target processes. For example, diffusion of reactive species in the target seems to play a key role in the time dependency of the poisoning process. The second obstacle is one of the classical problems with modeling: finding reliable parameters. For a large number of target/reactive gas combination there is a lack of fundamental parameters such as incorporation coefficients and sputter yields. If the thin film community wishes to obtain a predictive and quantitative understanding of reactive sputtering, this information is a necessity. This sounds pessimistic, but actually it is also an opportunity to keep sputter deposition of thin films a vibrant and active field [1]. 


\section{Acknowledgments}

This paper is a collective effort by all current members of the research group DRAFT who have used the RSD code to investigate a specific case study. As their contribution is equal, their names are put in random order, except for the first, second and last author. Special credits go to the second author who have pushed the RSD model further to include more tools, and to improve the description of the target processes. The authors also wish to thank all previous team members who, by experiments and stimulating ideas, enabled to seek for the limits of the RSD model.

\section{References}

[1] J. E. Greene, Review article: Tracing the recorded history of thin-film sputter deposition: From the 1800s to 2017, Journal of Vacuum Science \& Technology A 35 (5) (2017) 05C204. doi:10.1116/1.4998940.

[2] K. Strijckmans, D. Depla, A time-dependent model for reactive sputter deposition, Journal of Physics D: Applied Physics 47 (23) (2014) 235302. doi : $10.1088 / 0022-3727 / 47 / 23 / 235302$.

[3] K. Strijckmans, Modeling the reactive sputtering process, Thesis, Ghent University (2015).

URL http://hdl . handle.net/1854/LU-6961940

[4] K. V. Aeken, S. Mahieu, D. Depla, The metal flux from a rotating cylindrical magnetron: a monte carlo simulation, Journal of Physics D: Applied Physics 41 (20) (2008) 205307. doi:10.1088/0022-3727/41/ $20 / 205307$. 
[5] D. Depla, W. Leroy, Magnetron sputter deposition as visualized by monte carlo modeling, Thin Solid Films 520 (20) (2012) 6337 - 6354. doi:10.1016/j.tsf.2012.06.032.

[6] K. Strijckmans, D. Depla, RSD2013 v3/SIMTRA v2.2 - Simulation files of the examples in Modeling reactive magnetron sputtering: opportunities and challenges, Mendeley Data V1 (2019). doi:10.17632/ $235 \mathrm{y} 5 \mathrm{c} 368 \mathrm{v} \cdot 1$.

[7] K. Strijckmans, R. Schelfhout, D. Depla, Tutorial: Hysteresis during the reactive magnetron sputtering process, Journal of Applied Physics 124 (24) (2018) 241101. doi:10.1063/1.5042084.

[8] D. Depla, S. Mahieu, Reactive Sputter Deposition, Springer Series in Materials Science, Springer Berlin Heidelberg, 2008.

[9] S. Berg, T. Nyberg, Fundamental understanding and modeling of reactive sputtering processes, Thin Solid Films 476 (2) (2005) 215-230. doi:10.1016/j.tsf.2004.10.051.

[10] D. Depla, R. De Gryse, Target voltage measurements during dc sputtering of silver in a nitrogen/argon plasma, Vacuum 69 (4) (2003) 529-536. doi:10.1016/s0042-207x(02)00602-4.

[11] D. Depla, A. Colpaert, K. Eufinger, A. Segers, J. Haemers, R. De Gryse, Target voltage behaviour during dc sputtering of silicon in an argon/nitrogen mixture, Vacuum 66 (1) (2002) 9-17. doi:10.1016/ s0042-207x (01) 00415-8. 
[12] R. Schelfhout, K. Strijckmans, D. Depla, Anomalous effects in the aluminum oxide sputtering yield, Journal of Physics D: Applied Physics 51 (15) (2018) 155202. doi:10.1088/1361-6463/aab321.

[13] R. Schelfhout, K. Strijckmans, D. Depla, The existence of a double s-shaped process curve during reactive magnetron sputtering, Applied Physics Letters 109 (11) (2016) 111605. doi:10.1063/1.4962958.

[14] SiMTRA and RSD2013 can be downloaded from www.draft.ugent.be. The input files for the simulations presented in this paper will be available on the same website. (2018).

[15] W. D. Westwood, Calculation of deposition rates in diode sputtering systems, Journal of Vacuum Science \& Technology 15 (1) (1978) 1-9. doi:10.1116/1.569429.

[16] K. Strijckmans, D. Depla, Modeling target erosion during reactive sputtering, Applied Surface Science 331 (2015) 185 - 192. doi:10.1016/j. apsusc.2015.01.058.

[17] F. Cougnon, K. Strijckmans, R. Schelfhout, D. Depla, Hysteresis behavior during facing target magnetron sputtering, Surface and Coatings Technology 294 (2016) 215 -219. doi:10.1016/j. surfcoat.2016.03. 096.

[18] D. Depla, S. Mahieu, R. De Gryse, Magnetron sputter deposition: Linking discharge voltage with target properties, Thin Solid Films 517 (9) (2009) 2825-2839. doi:10.1016/j.tsf.2008.11.108.

URL < GotoISI> : / WOS: 000264331500001 
[19] F. Milde, G. Teschner, C. May, Gas inlet systems for large area linear magnetron sputtering sources, in: 44th Annual Technical Conference Proceedings, Society of Vacuum Coaters, 2001, pp. 204-209.

[20] J. Xia, W. Liang, Q. Miao, D. Depla, On the influence of local oxygen addition on the growth of sputter deposited yttrium oxide thin films, Surface and Coatings Technology 357 (2019) 768 - 773. doi:10.1016/ j.surf coat.2018.10.081.

[21] D. Depla, J. Haemers, R. D. Gryse, Influencing the hysteresis during reactive magnetron sputtering by gas separation, Surface and Coatings Technology 235 (2013) 62 -67. doi:10.1016/j.surfcoat.2013.07. 012.

[22] R. Tonneau, P. Moskovkin, A. Pflug, S. Lucas, Tio ${ }_{x}$ deposited by magnetron sputtering: a joint modelling and experimental study, Journal of Physics D: Applied Physics 51 (19) (2018) 195202. doi:10.1088/ 1361-6463/aabb72.

[23] A. Billard, C. Frantz, Low-frequency modulation of pulsed de or rf discharges for controlling the reactive magnetron sputtering process, Surface \& Coatings Technology 86-7 (1-3) (1996) 722-727. doi:10.1016/ s0257-8972(96) 03064-2.

[24] A. Billard, F. Perry, C. Frantz, Stable and unstable conditions of the sputtering mode by modulating at low frequency the current of a magnetron discharge, Surface \& Coatings Technology 94-5 (1-3) (1997) 345351. doi:10.1016/s0257-8972(97)00449-0. 
${ }_{430}[25]$ F. Perry, A. Billard, C. Frantz, An investigation of the pulse characteristics on deposition rate of reactively sputtered titanium dioxide films synthesised with a low-frequency modulation of the discharge current, Surface \& Coatings Technology 94-5 (1-3) (1997) 339-344. doi:10.1016/s0257-8972(97)00457-x. 